\title{
Molecular Basis for Membrane Selectivity of Antimicrobial Peptide Pleurocidin in the Presence of Different Eukaryotic and Prokaryotic Model Membranes
}

Reza Talandashti ${ }^{1}$, Hamid Mahdiuni ${ }^{2}$, Majid Jafari ${ }^{1}$, Faramarz Mehrnejad ${ }^{1 *}$

1- Department of Life Science Engineering, Faculty of New Sciences and Technologies, University of Tehran, P.O. Box 14395-1561, Tehran, Iran

2- Bioinformatics Lab., Department of Biology, School of Sciences, Razi University, P.O. Box 67149-67346, Kermanshah, Iran

*. Corresponding author: mehrnejad@ut.ac.ir 
Further information related to the distance between peptide and lipid bilayers as a function of time, the 2D and 3D structure of cholesterol molecule and its distribution in the $\mathrm{RBC}$ membrane, the number of contacts between Lys residues and membrane models, the snapshots during trajectories for each peptide-membrane complex, The average of the hydrogen bonds between lipid head groups, and membrane thickness for the pure membrane and peptide-membrane systems as a function of time. 
(A)

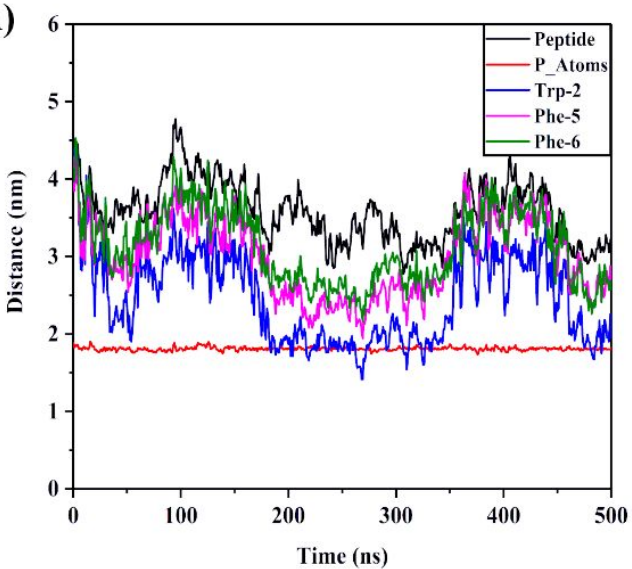

(C)

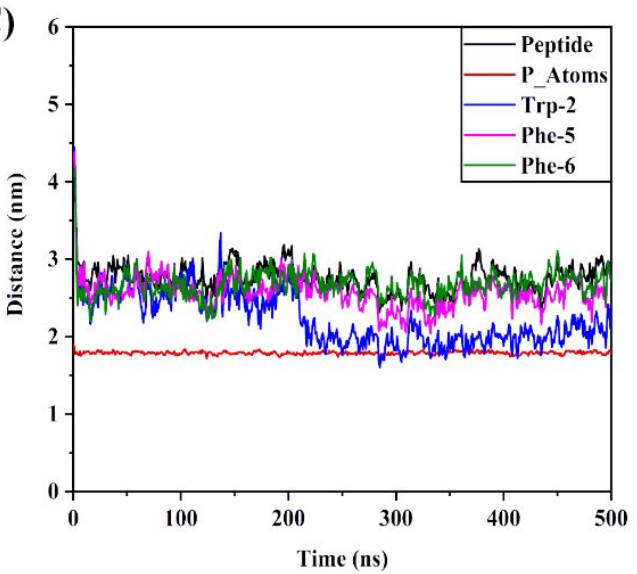

(E)

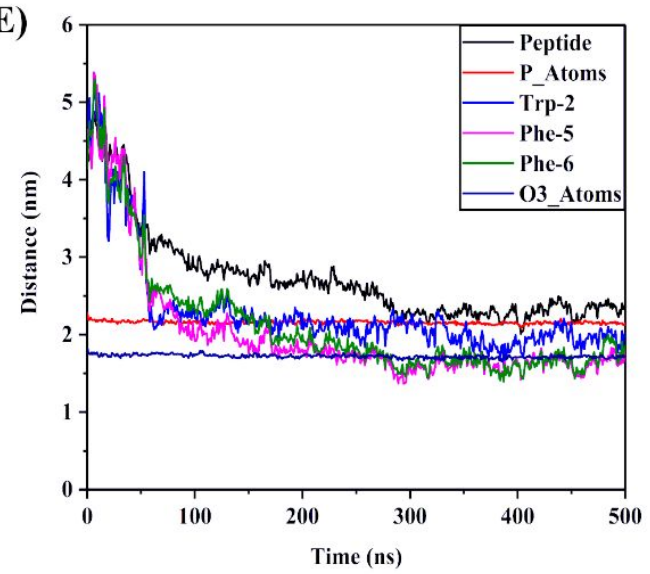

(B)

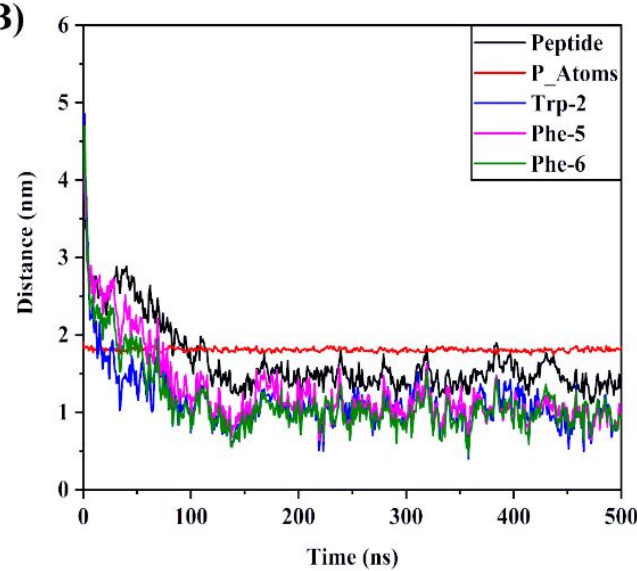

(D)

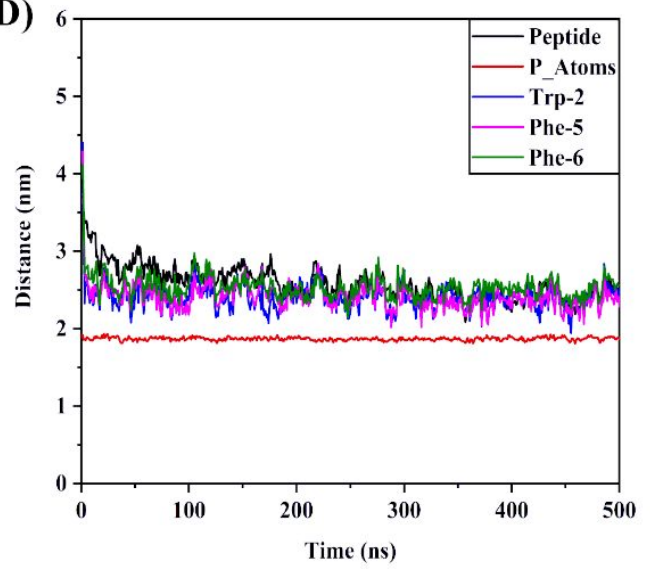

Fig S1. Represents the distance of the peptide from the membrane surface as the function of time in the (A) SDP1, (B) SDP2, (C) SDP3, (D) SDP4, and (E) SDP5 simulations. 
(A)

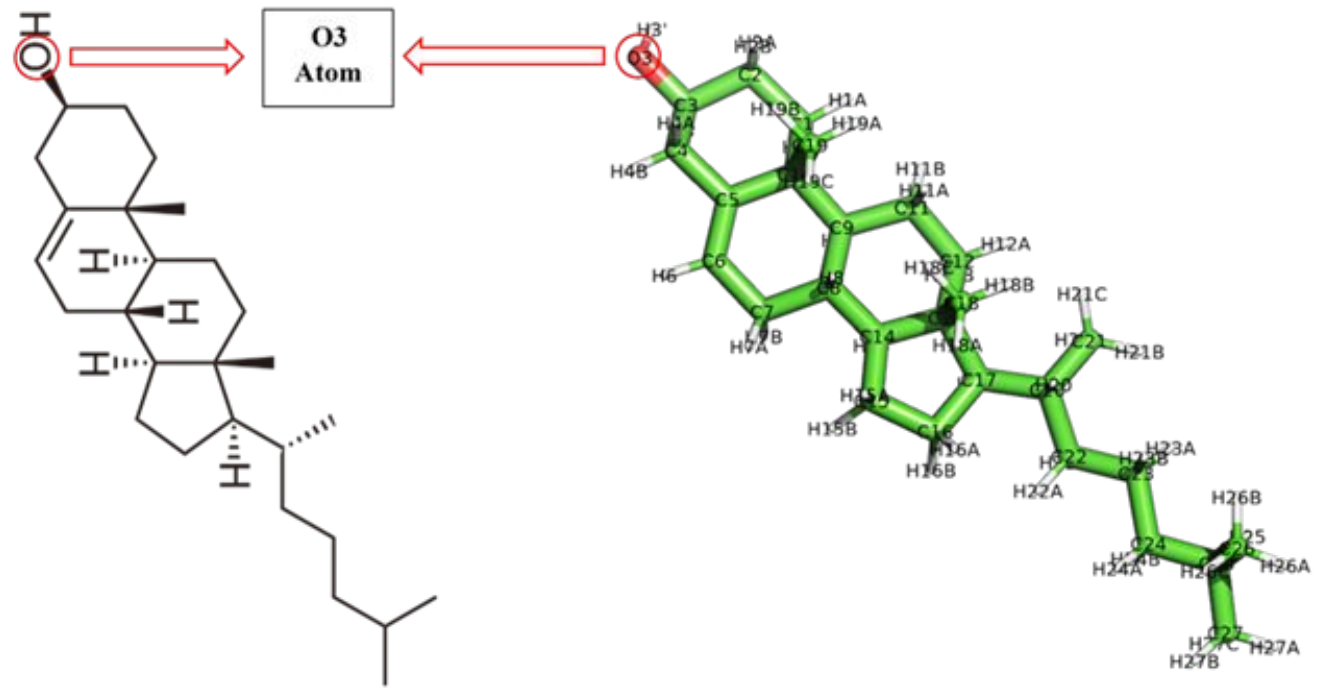

(B)

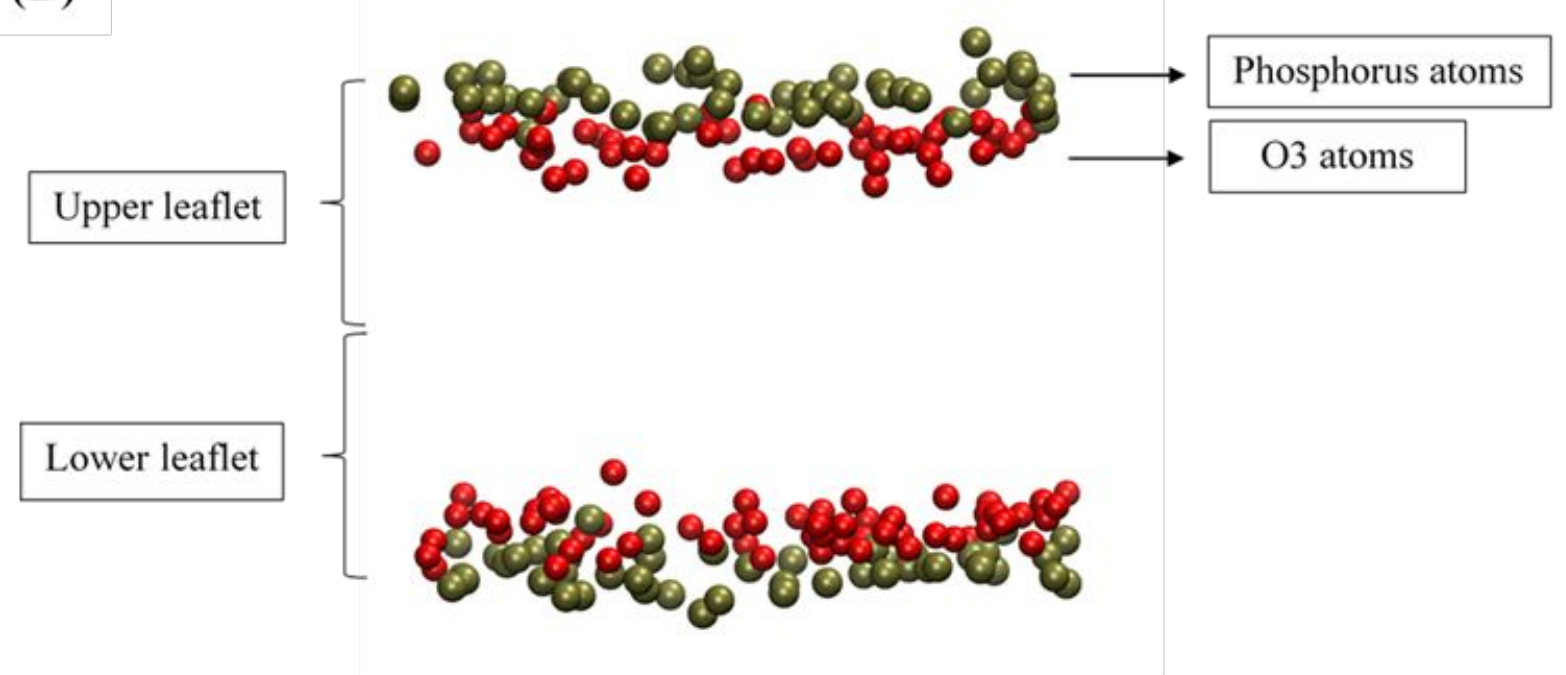

Fig S2. Represents (A) the 2D and 3D structure of cholesterol molecules and (B) its distribution in the RBC membrane. 


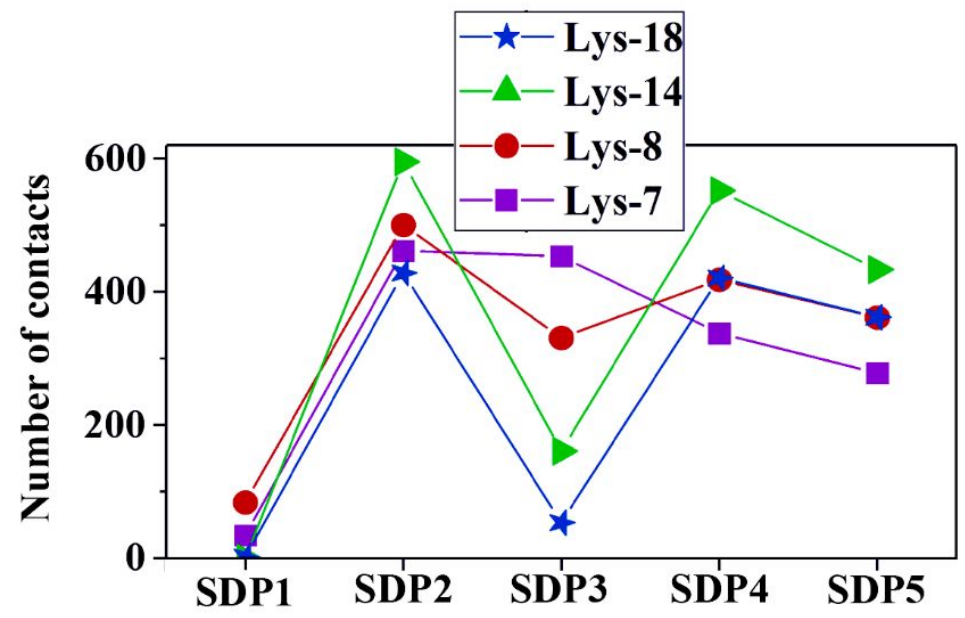

Figure S3. The number of contacts ( $>0.6)$ of the Lys residues in all peptide-membrane simulations. 


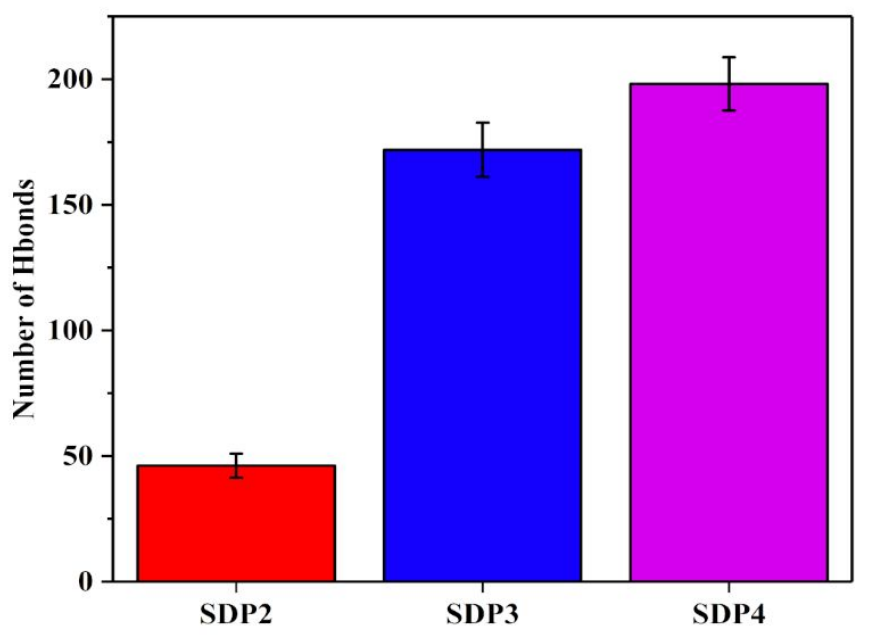

Figure S4. The average of the hydrogen bonds between lipid head groups in SDP2, SDP3, and SDP4 simulations. 


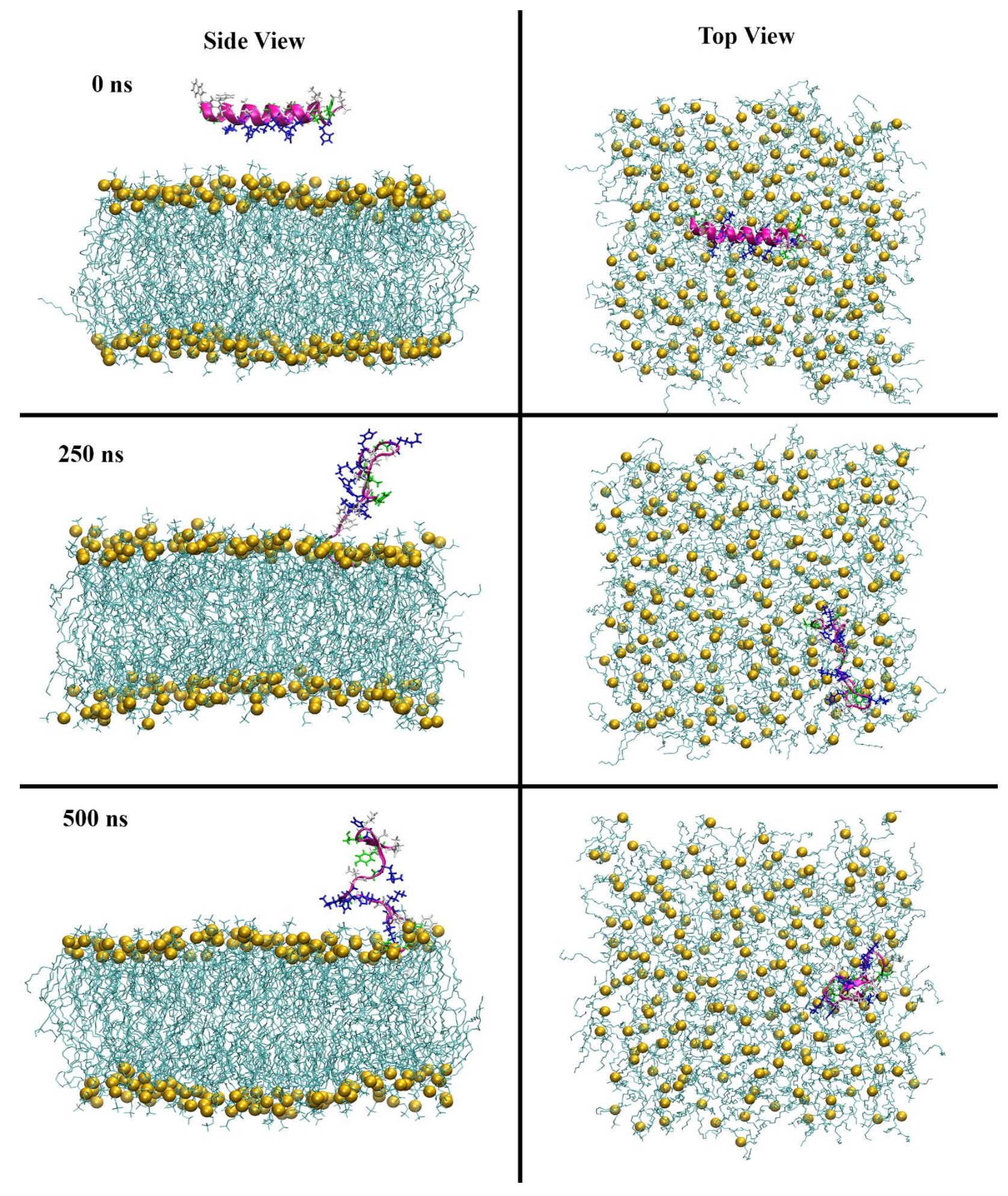

Figure S5. Represent the lateral and top view of the peptide-membrane complex in SDP1 system. The phosphorous atoms and lipid acyl chains were shown in yellow and cyanine color, respectively. The peptide backbone was shown in magenta and the side chain was colored based on the type of residue. 


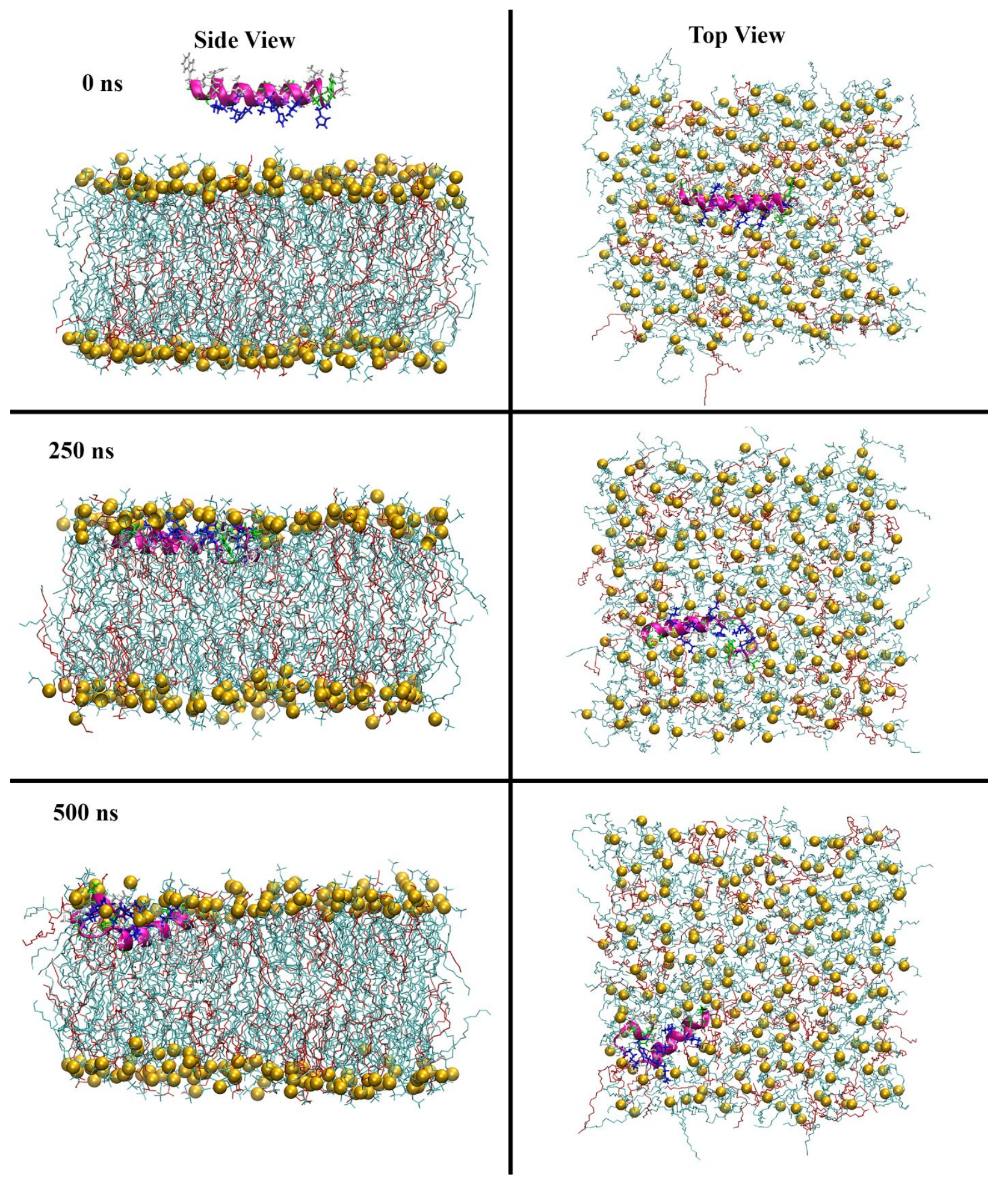

Figure S6. Represent the lateral and top view of the peptide-membrane complex in SDP2 system. The phosphorous atoms were shown in yellow color. The lipid acyl chains of the DOPC and DOPG were indicated in cyanine and red color, respectively. The peptide backbone was shown in magenta and the side chain was colored based on the type of residue. 


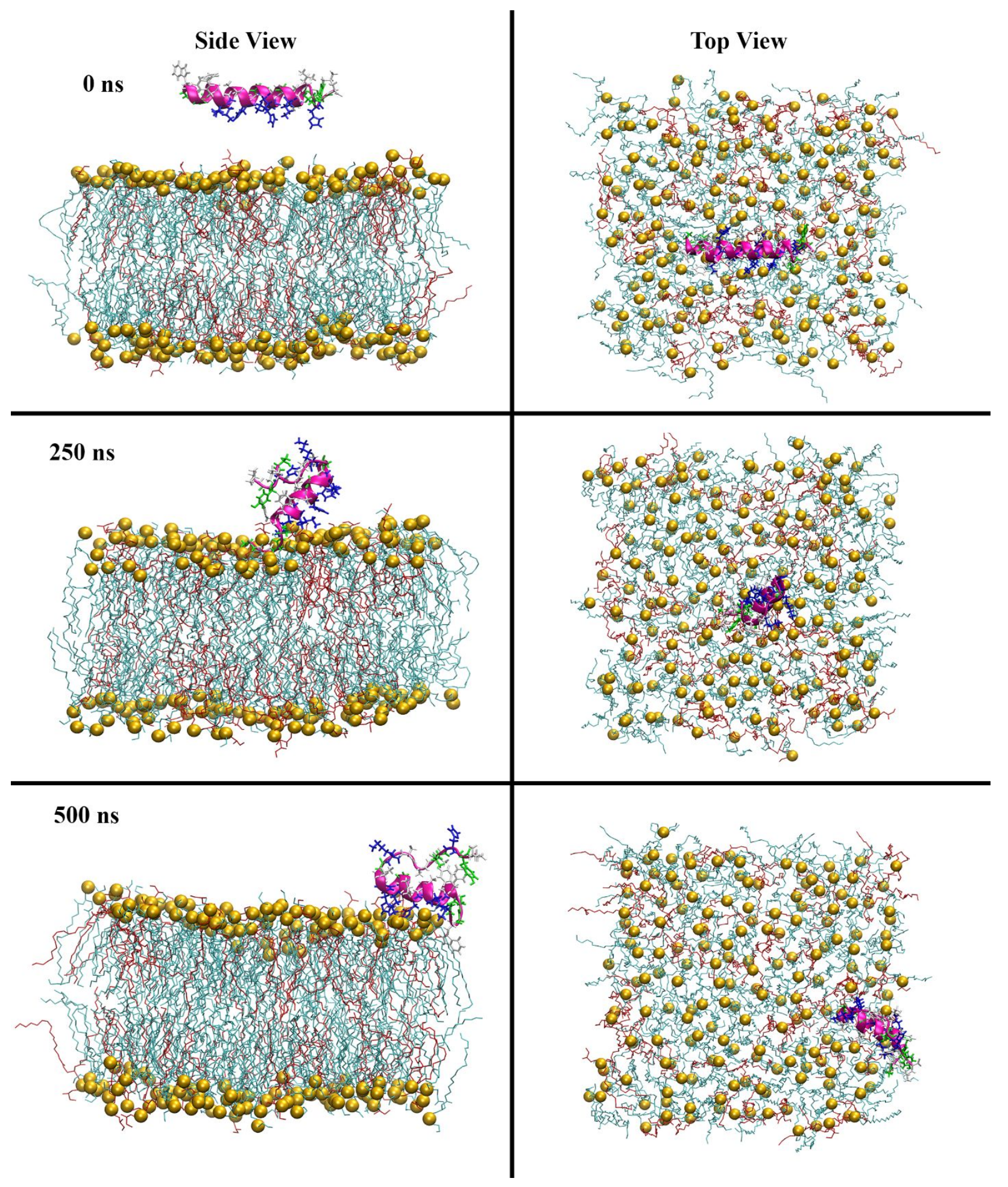

Figure S7. Represent the lateral and top view of the peptide-membrane complex in SDP3 system. The phosphorous atoms were shown in yellow color. The lipid acyl chains of the POPE and POPG were indicated in cyanine and red color, respectively. The peptide backbone was shown in magenta and the side chain was colored based on the type of residue. 


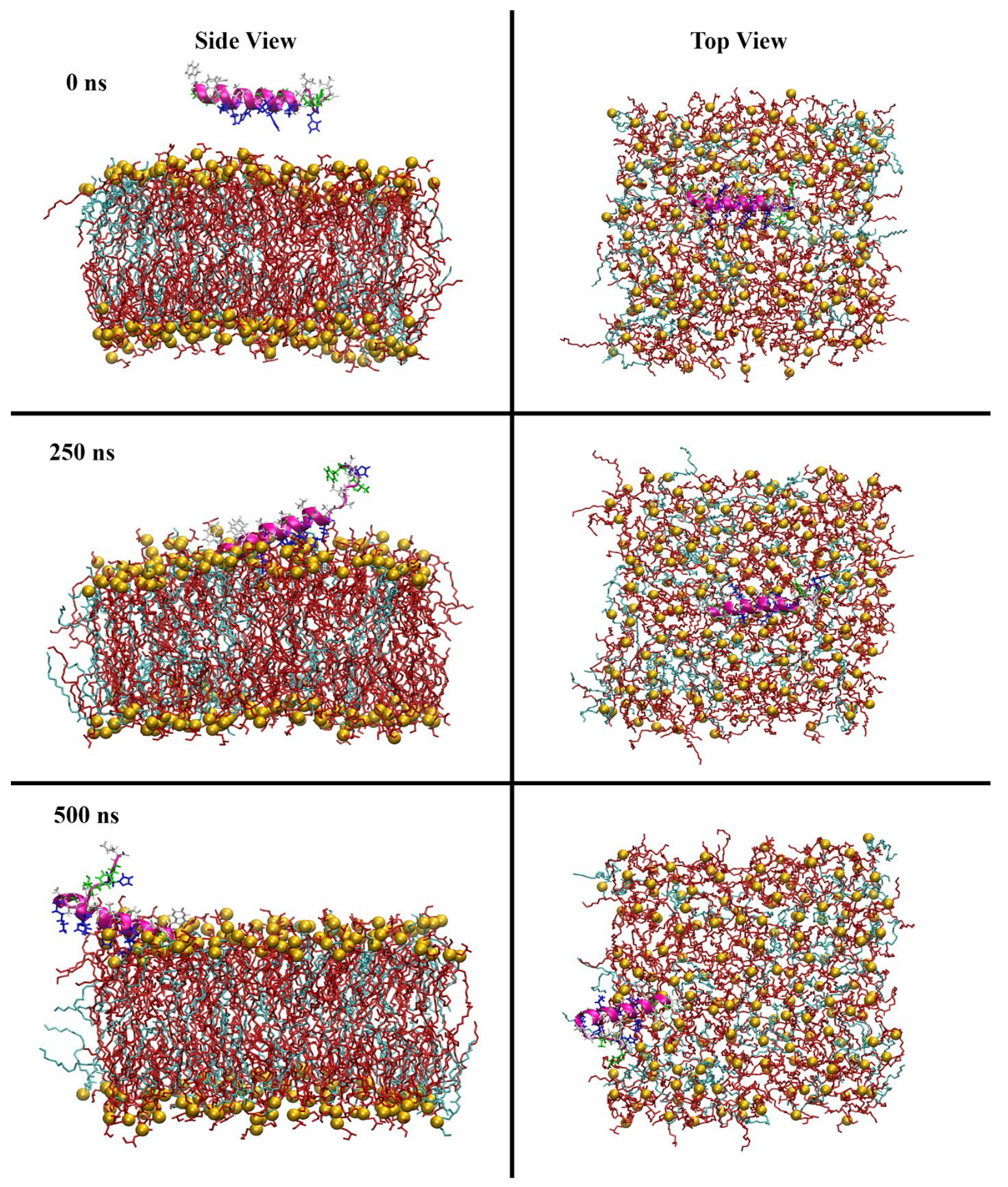

Figure S8. Represent the lateral and top view of the peptide-membrane complex in SDP4 system. The phosphorous atoms were shown in yellow color. The lipid acyl chains of the POPE and POPG were indicated in cyanine and red color, respectively. The peptide backbone was shown in magenta and the side chain was colored based on the type of residue. 


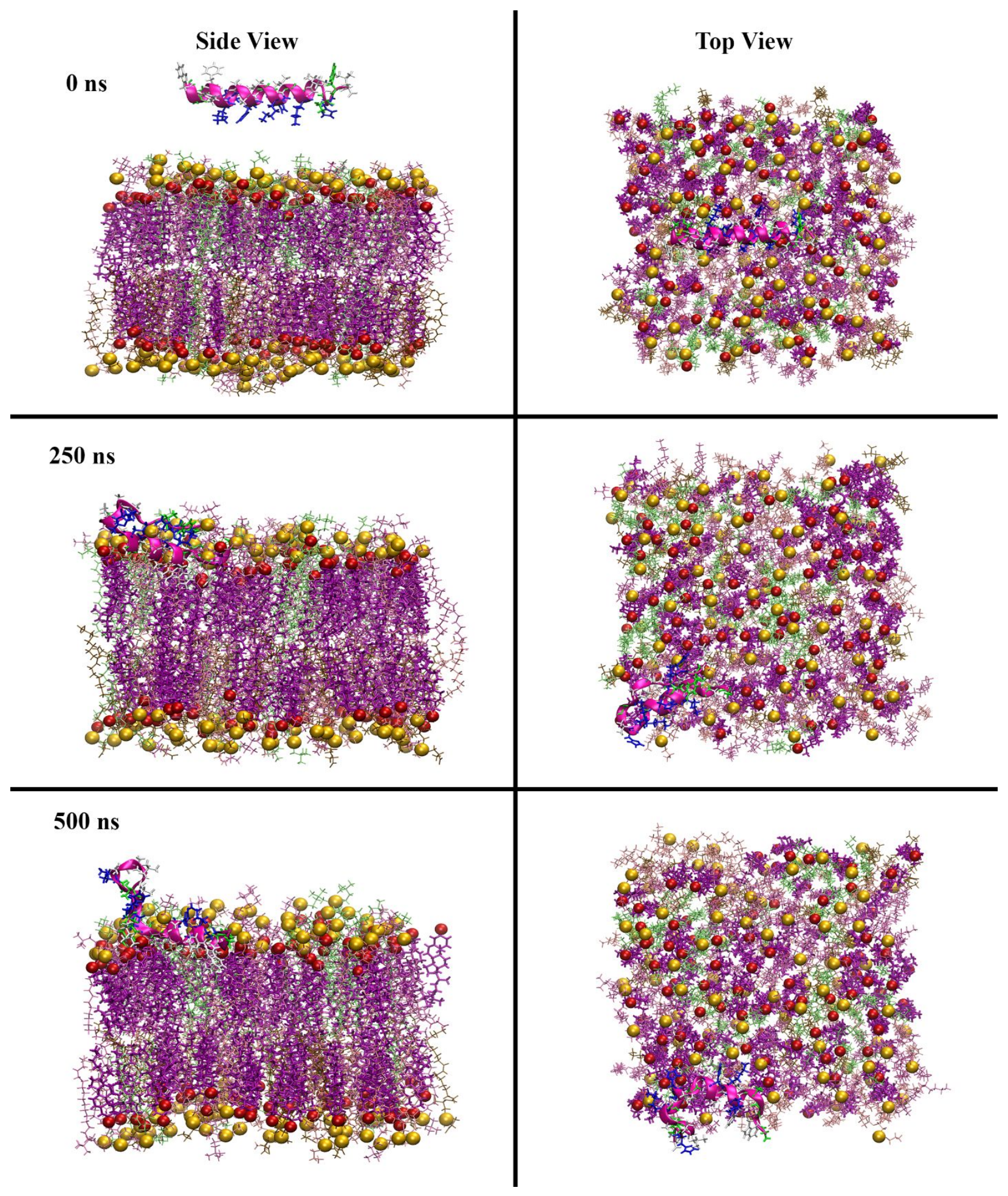

Figure S9. Represent the lateral and top view of the peptide-membrane complex in SDP5 system. The phosphorous and $\mathrm{O} 3$ atoms were shown in yellow and red color, respectively. The cholesterol molecules were indicated in purple color. The peptide backbone was shown in magenta and the side chain was colored based on the type of residue. 

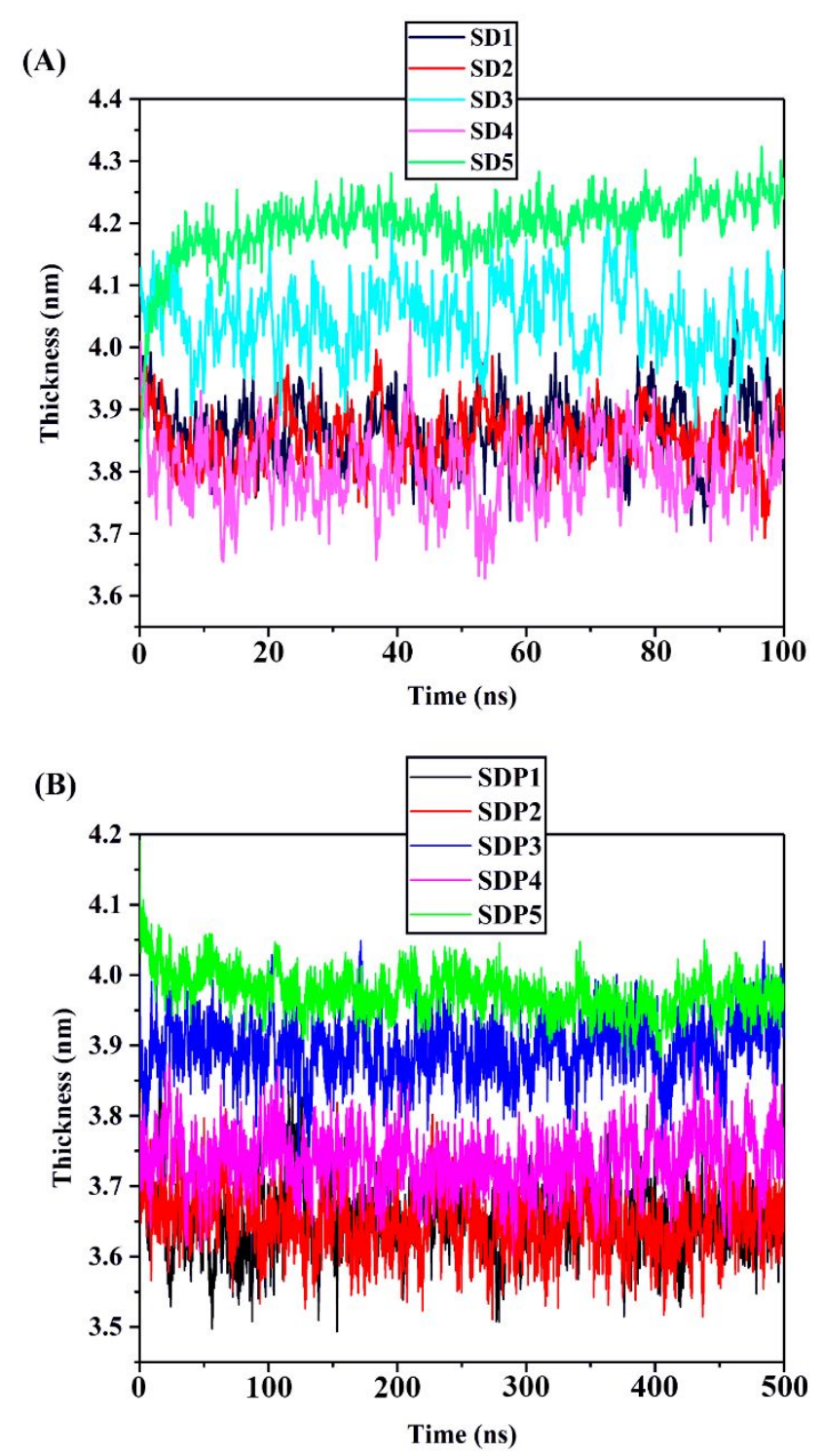

Figure S10. Represents the membrane thickness for (A) pure bilayers and (B) peptide-bilayer complex as the function of times. 